\title{
Multi Objective Data Aggregation Scheduling for Power Efficient WNSN
}

\author{
S.Madhavi ${ }^{1}$ and Tai Hoon Kim ${ }^{2}$ \\ Department of Computer Science and Engineering , PVP Siddhartha Institute of \\ Technology, Andhra Pradesh, India. \\ Department of Convergence and Security, Sungshin Womens University, 249-1- \\ Dongseon-dong 3-ga-Seoul, 136-742, Republic Korea
}

\begin{abstract}
A nano sensor can be charged using nano batteries. Various methods like energy harvesting had evolved to optimize the power utilization. These methods are helpful to prolong the network life time. Finding the optimum combination of parameters like maximizing the network lifetime from the limited available battery source and minimizing the noise in the channel for minimizing the energy consumption is a challenging task. We model this problem as a multi objective function problem and proposed a data aggregation scheduling method which uses the genetic algorithm. The results prove that this method slightly outperforms the method in [17].
\end{abstract}

Keywords: WNSN, Bosonic, Data Aggregation, Nanoscale communication, Base Station, Genetic Algorithm.

\section{Introduction}

With recent advancements in nanotechnology, energy consumption techniques for wireless nano-scale sensor networks (WNSNs) have become many researchers topic. Since battery power and bandwidth are the two limited resources available in WNSNs recharging the nanosensors is a great challenging task for many researchers today. Many researchers had proposed energy harvesting methods to power a nanosensor since it is very difficult to deploy batteries in nanosensors. Since the nanosensors have to aggregate data to the base station, increasing network life time with minimum energy consumption is the major research interests of many researchers today. Hence in this paper we propose the model for the multi objective function for finding an optimal solution which is a optimum combination of these variables.

The rest of paper is organized as follows. In the next section, we introduce the background for the communication model in WNSNs. In Section III we introduce our optimization problem. Simulation results are presented in Section IV, and the conclusions in Section V.

\section{Background and Related Work}

Operating in the $\mathrm{THz}$ frequency allows nanosensors to consume low energy while providing connectivity in the nano to milli meter communication range [8]. The nodes use pulse based communication and Rate Division Time Spread On-Off Keying (RD TSOOK) [8] as the modulation mechanism. Wireless Nano-scale Sensor Networks (WNSNs) can operate over terahertz band ranging from $0.1-10 \mathrm{THz}$ and this $\mathrm{THz}$ band enables nanosensors to consume low energy. And this is reason how nanosensors could detect nanoparticles like hazardous elements in the atmosphere. Since the WNSNs can sense and control important physical processes at the molecular level these are used to enhance the performance of many chemical and biological processes $[3,15,16]$. 
In [15] Nanosensors are tiny devices made from novel nano materials capable of sensing new types of physical, chemical and biological phenomenon at the molecular level [13]. These are used in many application areas like biology, medicine, chemistry, military, industrial and online microscopic environmental monitoring. Since the nanosensors have to aggregate data to the base station energy consumption methods plays a vital role in WNSN. In [1] the authors proposed a bosonic network model for communication where an optimal schedule through utilizing the observable or measurable physical properties the network topology is suggested.

Several parameters are introduced in the modelling of energy harvesting and consumption that can affect the optimum energy utilization of a nanonode like using the optimum values for code weight, repetition and packet size[14]. Jornet and Akyildiz proposed a energy harvesting and consumption processes in WNSNs [4]. Jornet and Akyildiz [4] argue that using code weight saves energy in transmission since the lower the code weight, the lower the energy for transmission. Doan Duc Tung, Fabio Mottola, Alfredo Testa proposed a method for minimizing power losses in distribution systems using GA in planning and operation. Shipeng Du, Qianzhi Shao and Gang Wang analysed DG influences on system losses in distribution network. Hong Liu, Yinchang Guo, Shaoyun Ge, and Mingxin Zhao studied on the impact of DG configuration on maximum use of load supply capability in distribution power systems. Zeng Pin-zhuo, Wang Ke-you, Li Guo-jie and Jiang Xiu-chen proposed a method for optimization of distributed generation integrated into micro grids considering the correlation of DGs.

Xingming Sun1, Hongwei Qian, Baowei Wang, Qi Liu proposed an enhanced data collection protocol based on CTP. Aijun Zhang, Xingfeng Xiao, Xuan Fu, Dan Wang od on new energy power grid-integration on grid power quality. Chen Wei , Jing Rui , Zhou Wen, LIN Chaoran proposed an innovation network structure of industrial cluster of new energy vehicles in the northeast China. Jinsong Liu studied on the development strategies of new energy automotive industry based on car charging stations and battery management. In this paper we propose a method which is an extension to our method proposed in [17] where the optimal number of concurrent neighbors are obtained.

Generally the radio communication in the terahertz band is affected by the chemical compositions of the channel in two different ways[13]. Like the radio signal is attenuated because molecules in the channel absorb energy in certain frequency band and this absorbed energy is re-radiated by the molecules and create noise in the channel. Using these properties the scheduling is proposed and the new schedule improved the network life time.

\section{Proposed Optimized Model.}

Since nano-sensors have extremely limited energy storage capacity, finding a solution to design simple and energy efficient modulation and coding schema are considered a major challenge for the researchers. Our optimization model for minimizing energy consumption is proposed as a multi objective function and is different from previous works. Earlier several methods are proposed like minimizing the energy consumption through adapting the modulation, efficient routing protocols, or adaptive duty cycle of MAC, packet size and code weight for optimization.

Our model introduces the concept of optimizing several communication objective functions simultaneously, by applying the GA method. In our model we take into account the communication energy consumption for both transmitter and receiver, where the noise produced by the concurrent transmitters is also considered.

Consider a wireless sensor network with $\mathrm{n}$ arbitrarily distributed nanosensor nodes. A node can act as a cluster head and is adaptive by adjusting the transmission powers based on the communication distance. Each sensor node aggregates data to the base station. The network is divided into clusters and each cluster head aggregates the data to the base 
station. Every node forwards data to an optimal cluster head. For data aggregation in this paper we proposed a novel solution for improving the performance of WNSN. It consists of the following three steps:

1. Identification of ConcHead Nodes

2. Constructing a schedule with a list of neighbours of ConcHeads.

3. Finding an optimal schedule.

The optimal cluster head is elected using the following equations

The identification of cluster head contains two phases

\section{Identification of Cluster Head Nodes}

Repeat

1. Repeat the following for $\mathrm{i}=1$ to $\mathrm{n}$ nodes in the network

2. For each node $\mathrm{Vj} j=1$ to $\mathrm{n}$ where $\mathrm{i}$ not equal to $\mathrm{j}$ in the network

3. Find a set of all nodes in the network that can aggregate data to a node $\mathrm{Vi}$ in a time slot tk. Call Find(Vi,Vj)

4. Let $\mathrm{T}(\mathrm{Vi})$ represents the total number of nodes that can aggregate data to $\mathrm{Vi}$

5. Constructing a schedule with a list of neighbors' of ConcHeads.

6. Find a node that has maximum in degree that is $\mathrm{T}(\mathrm{Vx})$, Call it the cluster head for slot $\mathrm{k}$

7. Increment $k$ by 1

until network contains at least two nodes

To find a set of all nodes in the network that can aggregate data to a node $\mathrm{Vi}$ in a time slot tk. Find(Head, Node)

1. $\quad \mathrm{V}$ is the set of $\mathrm{n}$ ubiquitous nodes and each node can send and receive data in the maximum range crmax where the transmission power of a node is always $\leq$ crmax,

2. Let $\mathrm{Vj}$ denote a node that can transmit concurrently with node $\mathrm{Vi}$ as the head. For a successful transmission the SINR perceived at the receiver should be greater than or equal to $\beta$ i.e.

$$
\frac{p_{s} / d_{s}^{\alpha}}{N 0+\sum_{j=1}^{c o n c} P_{r} / d_{j}^{\alpha}} \geq \beta \rightarrow 1
$$

3. Let Ps and Pr denote the transmission powers of sender and receiver nodes respectively. da is the distance between sender and receiver nodes and dj is the distance between a concurrent transmitter and the receiver. $\alpha$ is the path loss ratio, which has a typical value between 2 and 3 . N0 is the ambient noise.

4. $\beta$ is the threshold for a successful transmission. The transmission of becomes successful iff the SINR perceived at the receiver is greater than or equal to a threshold value $\beta$.

5. Generally the radio communication in the terahertz band is affected by the chemical compositions of the channel in two different ways[13].

1. The radio signal is attenuated because molecules in the channel absorb energy in certain frequency bands.

2. This absorbed energy is re-radiated by the molecules and create noise in the channel.

Our proposed model considers these two effects [13].

Let the radio channel is a medium consisting of $\mathrm{N}$ different chemical species $\mathrm{S}_{1}, \mathrm{~S}_{2} \ldots$ $\mathrm{S}_{\mathrm{N}}$. 
Let the effect of each chemical species $\mathrm{Si}$ on the radio signal is characterized by its molecular absorption coefficient $\mathrm{K}_{\mathrm{i}}(\mathrm{f})$ at frequency $\mathrm{f}$.

Let $m_{i}$ be the mole fraction of chemical species $S_{i}$ in the medium.

The medium absorption coefficient $\mathrm{K}(\mathrm{f})$ at frequency $\mathrm{f}$ determines the attenuation and the molecular absorption noise in the radio channel for different molecules in different frequencies ie

$K\left(t_{\mathrm{f})=} \sum_{i=1}^{N} m_{i}(t) K_{i}(f) \rightarrow 2\right.$

And is obtained from the weighted sum of the molecular absorption coefficients in the medium:

Hence the total attenuation, i.e. attenuation due to spreading and attenuation due to molecular absorption, at frequency $\mathrm{f}$ and a distance $\mathrm{d}$ from the transmitter is given by [11]:

$$
A(f, d)=\left(\frac{4 \pi f d}{c}\right)^{2} \times e^{(K(f) \times d)} \rightarrow 3
$$

where $\mathrm{c}$ is the speed of light in the vacuum.

The power spectral density (PSD) of the received signal $\operatorname{Pr}(f, d)[11]$ at frequency $f$ and distance $\mathrm{d}$ is:

$P_{r}(f, d)=\frac{U(f)}{A(f, d)} \rightarrow 4$

where $\mathrm{U}(\mathrm{f})[11]$ is the PSD of the transmitted signal at frequency $\mathrm{f}$. The average received energy at distance $\mathrm{d}$ is:

$$
E_{r}(d)=\int_{B} P_{r}(f, d) T_{p} d f \rightarrow 5
$$

where $\mathrm{Tp}$ is the duration of the transmitted pulse in second and B is the bandwidth.

And Equation 1 is modified as

$$
\operatorname{SINR}(t, f, d)=\frac{U(t, f)}{A(t, f, d) N_{a b s}(t, f, d)}
$$

Where

Nabs is the molecular absorption noise is due to the re radiation of absorbed radiation by the molecules and it is given by is due to

$$
N_{a b s}(t, f, d)=k_{B} T_{0}\left(1-\exp \left(-K(t, f)^{*} d\right)\right)
$$


The Noise factor is calculated using the equations 1 to 7 . Every node tunes itself to an optimal power, in such a way that the SINR perceived at the receiver should be greater than or equal to $\beta$.

End

Finding an optimal schedule.

Various methods are used to solve multi objective optimization problems, such as the method of objective weighting or min/max formulation [14], to merge multiple objectives into one objective so that the resulting solution depends mainly on the weight vector assigned to each objective [14]. We would like to use a GA to optimize the number of clusters and sensor connections for an arbitrary network. Once cluster-heads are selected, each regular node connects to its nearest cluster-head. Each node in a network is either a cluster-head or a "member" of a cluster-head. Each regular node can only belong to one cluster-head. Each cluster-head collects data from all sensors within its cluster and each head directly sends the collected data to the sink. Therefore, GA is used to determine optimal number of $\mathrm{CHs}$ and their locations by minimizing the following objective function $\mathrm{F}(\mathrm{X})[17]$ :

$F(X)=w\left(\frac{E_{\text {disp }}}{E_{\text {live }}}\right)+(1-w)\left(\frac{L}{N_{\text {live }}}\right) \rightarrow 6$

Where $\mathrm{E}_{\text {live }}$ is the total energy for all live nodes in sensor field. It is described by

$E_{\text {live }}=\sum_{j=1}^{N_{\text {live }}} E_{o}(j)=N_{\text {live }} E_{o} \rightarrow 7$

And $\mathrm{E}_{\mathrm{disp}}$ is the total dissipated energy of all live nodes in the sensor field.

Inorder to select the optimal node as a cluster head the genetic algorithm is applied.

Initially the senor network is assumed to be a set of $\mathrm{N}$ nodes and with all the edges $\mathrm{E}$.

As in [17] initialize by defining the parameters of the energy model and repeat the following steps until the stopping criteria is met..

Cluster Heads are elected as follows

1. Apply Genetic algorithm by setting $\mathrm{P}_{\mathrm{s}}$ initial bits binary chromosome

2. Calculate the objective function for all Chromosomes

3. Find the data aggregation schedules using the equations 1 to 5 and find the optimal schedule for it using the equations 1 to 7 .

4. Find the best chromosomes based on the fitness function $1 / \mathrm{F}(\mathrm{x})$ and using roulette wheel selecton algorithm using equations 6-7

5. From each pair of parent chromosomes apply crossover operation based on the rate of crossover

6. Apply mutation to all genes of each child based on the probability of the mutation rate

7. Calculate the objective function for the new chromosomes

8. Select the best chromosome and let the children be the parents for the next generation population pool

\section{Results}

We assumed 100 homogenous nodes with initial energy of $0.5 \mathrm{~J}$ scattered randomly within a $100 \times 100 \mathrm{~m}^{2}$ sensor field. The BS was positioned at point $(50,300) \mathrm{m}$ and the packets sent were 2000 bit plus 50 bit control packets. The GA parameters are set as Ps= 
50 and $\mathrm{pm}=0.11$ and $\mathrm{pc}=0.7$ weightining facor $\mathrm{w}$ is 0.95 and maximum number of generation is 150. In [17] the first node died after 1184 rounds ad all nodes died after 1592 rounds. The number of selected $\mathrm{CHs}$ in each round changes from 1 to 5 . The proposed protocol slightly outperforms [17] like the first node died after 1294 rounds and all nodes died after 1704 rounds.

\section{Conclusions}

The proposed method considers optimization model for minimizing energy consumption in WNSN. Our model uses a objective function optimization and a GA method. In our model we take into account the communication energy consumption for both transmitter and receiver and the noise produced by the concurrent transmitters.

We considered that the radio signal is attenuated because molecules in the channel absorb energy in certain frequency bands and this absorbed energy is re-radiated by the molecules and create noise in the channel. By considering the above principle in finding the number of concurrent transmitters simultaneously applying the GA we increased the network lifetime.

\section{References}

[1] I. F. Akyildiz and J. M. Jornet, "Electromagnetic wireless nanosensor networks," Nano Comm. Networks, vol. 1, no. 1, (2010), pp. 3-19.

[2] I.F. Akyildiz, F. Brunetti, and C. Blazquez, "Nanonetworks: A new communication paradigm," Computer Networks, vol. 52, no. 12, (2008), pp. 2260-2279.

[3] S. Cui, A. Goldsmith, and A. Bahai, "Energy-constrained modulation optimization," Wireless Communications, IEEE Transactions on, vol. 4, no. 5, (2005), pp. 2349-2360.

[4] J. Jornet and I. Akyildiz, "Joint energy harvesting and communication analysis for perpetual wireless nanosensor networks in the terahertz band," IEEE Trans. on Nanotechnology, vol. 11, (2012), pp. 570580.

[5] T. van Dam and K. Langendoen, "An adaptive energy-efficient mac protocol for wireless sensor networks," in Proceedings of ACM SenSys, (2003), pp. 171-180.

[6] W. Heinzelman, A. Chandrakasan, and H. Balakrishnan, "Energyefficient communication protocol for wireless microsensor networks", in System Sciences, 2000. Proceedings of the 33rd Annual Hawaii International Conference, vol.2, (2000), p. 2.

[7] J. Jornet and I. Akyildiz, "Low-weight channel coding for interference mitigation in electromagnetic nanonetworks in the terahertz band," in IEEE Intl. Conference on Comm. (ICC), (2011), pp. 1 -6.

[8] J. M. Jornet and I. F. Akyildiz, "Information capacity of pulse-based wireless nanosensor networks," in IEEE SECON, (2011), pp. 80-88.

[9] K. Deb, "Multi-objective optimization," in Search Methodologies (E. Burke and G. Kendall, eds.), Springer US, (2005), pp. 273-316.

[10] S.-Y. Ni, Y.-C. Tseng, Y.-S. Chen, and J.-P. Sheu, "The broadcast storm problem in a mobile ad hoc network," in Proceedings of ACM MobiCom, (1999), pp. 151-162.

[11] J. Jornet and I. Akyildiz. "Channel modeling and capacity analysis for electromagnetic wireless nanonetworks in the terahertz band. IEEE Transactions on Wireless Communications", vol. 10, no. 10, (2011), pp. 3211-3221.

[12] K. Deb, S. Agrawal, A. Pratap, and T. Meyarivan, "A fast elitist nondominated sorting genetic algorithm for multi-objective optimization: NSGA-II," International Conference on Parallel Problem Solving from Nature (PPSN), (2000), pp. 849-858

[13] E. Zarepour, M. Hassan, C. T. Chou, A. A. Adesina, "Frequency Hopping Strategies for Improving Terahertz Sensor Network Performance over Composition Varying Channels

[14] S. Mohrehkesh and M. C. Weigle, "Optimizing Communication Energy Consumption in Perpetual Wireless Nanosensor Networks"

[15] E. Zarepour, A. A. Adesina, M. Hassan, and C. T. Chou. "Innovative approach to improving gas-toliquid fuel catalysis via nanosensor network modulation", Industrial and Engineering Chemistry Research, vol. 53, no. 14, (2014), pp. 5728-5736.

[16] E. Zarepour, A. A. Adesina, M. Hassan, and C. T. Chou. Nano Sensor Networks for Tailored Operation of Highly Efficient Gas-ToLiquid Fuels Catalysts. In the proceedings of Australasian Chemical Engineering Conference, Chemeca 2013, Brisbane, Australia, (2013).

[17] S. Madhavi , Tai honn Kim “ Energy Efficient Genetic inspired scheduling for data aggregation”, IJCA, vol. 9, no. 3, (2016), pp. 227-234. 
[18] D. D. Tung, F. Mottola and A. Testa “, Minimizing Power Losses in Distribution Systems Using GA in Planning and Operation: A case Study for DGs and SCs International Journal of Grid and Distributed Computing, vol.10, no.5, (2017), pp. 1-12

[19] S. Du, Q. Shao and G. Wang, "Analysis of DG Influences on System Losses in Distribution Network", International Journal of Grid Distribution Computing, vol. 8, no.5, (2015), pp. 141-152.

[20] H. Liu, Y. Guo, S. Ge, and M. Zhao, "Impact of DG Configuration on Maximum Use of Load Supply Capability in Distribution Power Systems", Hindawi Publishing Coporation - Journal of Applied Mathematics, (2014).

[21] Z. Pin-zhuo, W. Ke-you, L. Guo-jie and J. Xiu-chen, "Optimization of Distributed Generation Integrated into Micro Grids Considering the Correlation of DGs", International Journal of Grid Distribution Computing, vol 8, no.6, (2015), pp. 105-116

[22] X. Sun1, H. Qian, B. Wang, Q. Liu, "ECTP: An Enhanced Data Collection Protocol based on CTP", International Journal of Grid and Distributed Computing, vol.6, no.5, (2013), pp.83-92.

[23] A. Zhang, X. Xiao, X. Fu, D. Wang, "Research on New Energy Power Grid-Integration on Grid Power Quality", International Journal of Grid and Distributed Computing, vol. 9, no. 4, (2016), pp.1-12

[24] C. Wei , J. Rui, Z. Wen , L. Chaoran, "Innovation Network Structure of Industrial Cluster of New Energy Vehicles in the Northeast China", International Journal of Smart Home, vol. 10, no. 6, (2016), pp. $127-136$

[25] J. Liu, "Research on the Development Strategies of New Energy Automotive Industry Based on Car Charging Stations and Battery Management", International Journal of Smart Home, vol. 9, no. 7, (2015), pp. 213-222.

[26] S. Madhavi, "Secured Data Aggregation Scheduling in Ubiquitous Quantum Sensor Networks", Asiapacific Journal of Multimedia Services Convergent with Art, Humanities, and Sociology, vol.4, no.1, (2014), pp. 17-30.

\begin{abstract}
Authors
S.Madhavi, She received her M.S.degrees from the BITS , Pilani in 1999 and $\mathrm{PhD}$ in Computer Science and Engineering in 2011. She is presently working as a Professor in the department of Computer Science and Engineering, P.V.P. Siddhartha Institute of Technology, India. She published 20 papers and two edited books. Her research interests are Wireless Sensor Networks, Quantum Cryptography, Neural Networks and Fuzzy logic and Object Oriented Analysis and Design Patterns.
\end{abstract}

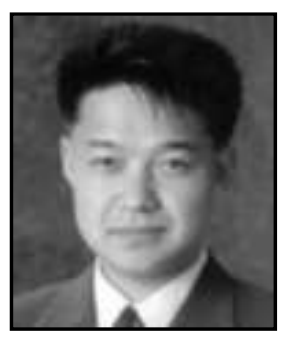

Tai Hoon Kim, He received B.E., and M.E., degrees from Sungkyunkwan University in Korea and and Ph.D. degrees from University of Bristol in UK and University of Tasmania in Australia. Now he is working for Department of Convergence Security, Sungshin W. University, Korea. His main research areas are security engineering for IT products, IT systems, development processes, and operational environments. He worked as researcher at Technical Institute of Shindoricoh, as a senior researcher at the Korea Information Security Agency, at the DSC (Defense Security Command), as a research professor at E-wha Woman University. He wrote many books about the software development, OS such as Linux and Windows 2000, and computer hacking \& security. He was a General Chair of ICHIT 2006, MUE 2007 and ISA 2008, Steering Committee Chair of FBIT 2007, IPC 2007, FGCN 2007 and MUE 2008, and Publicity Chair of JRS 2007. Now he is a Steering Committee Chair of FGCN 2008, ASEA 2008, SecTech 2008, BSBT 2008 and UNESST 2008. He was a Guest Editor of AJIT and FGCS Journal. 
International Journal of Control and Automation Vol.10, No.7 (2017) 